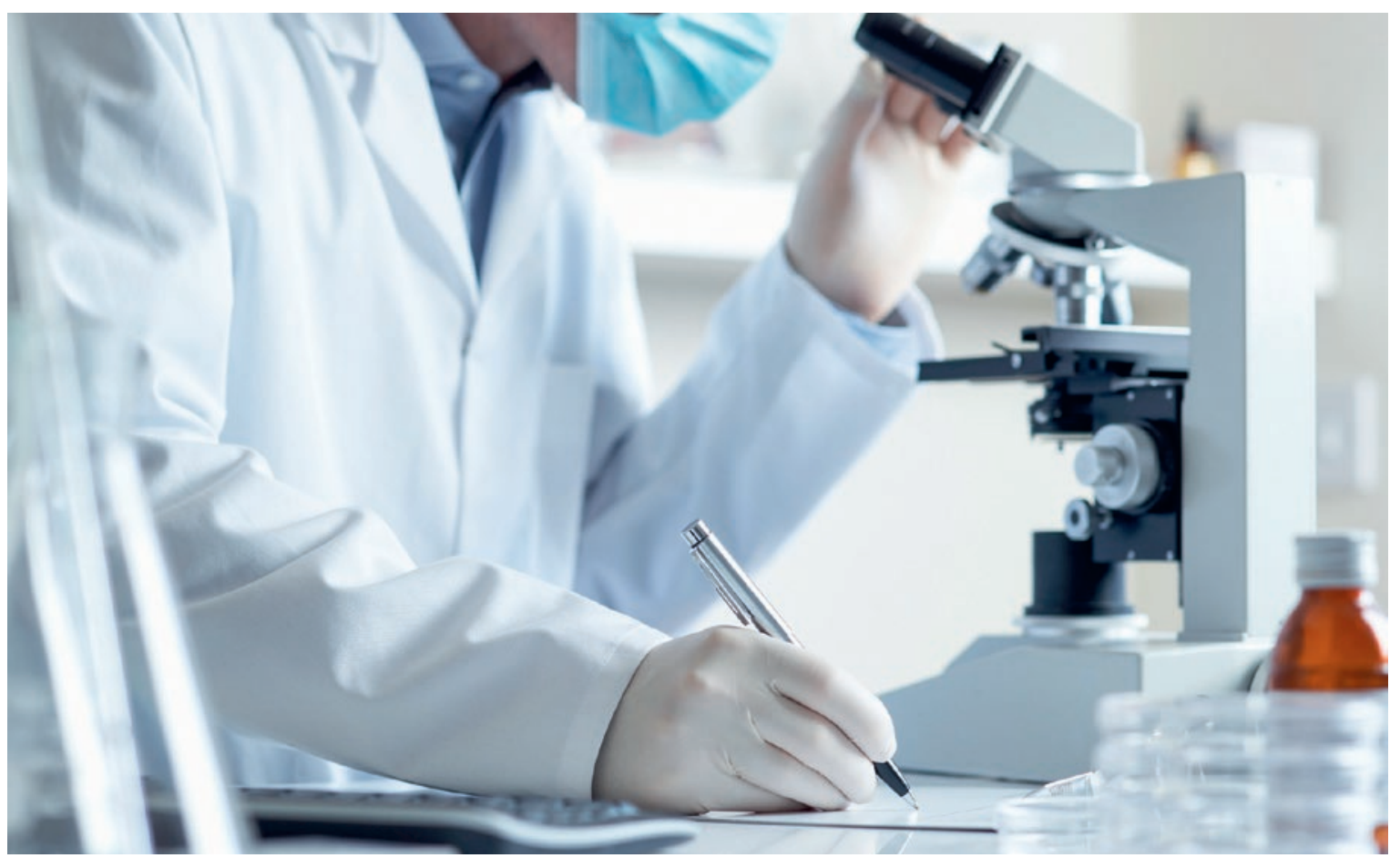

\title{
Der Generalkonsent aus Sicht der klinischen Forschung
}

\section{Martin Fey}

Prof. em. Dr. med., Senior Consultant, Universitätsklinik für medizinische Onkologie, Inselspital und Universität Bern

Speditive Forschung ermöglichen und die Rechte von Probandinnen und Probanden schützen - diesen verschiedenartigen Anforderungen wird der Generalkonsent zuverlässig gerecht. Der Autor stellt der Kritik am heutigen Modell des Generalkonsents Argumente aus der klinischen Forschung entgegen.

Das Humanforschungsgesetz (HFG SR 810.30) verlangt, dass Patienten oder Probandinnen, die für eine klinische Studie infrage kommen, vorab umfassend und verständlich über das Forschungsprotokoll aufgeklärt werden, sodass sie informiert und frei entscheiden können, ob sie mitmachen möchten oder nicht. Nun gibt es Projekte, die lediglich asserviertes klinisches Material (z. B. Biobanken, Radiologie-Bildarchive) auswerten möchten, das bei Routinetätigkeiten oder als Anhängsel eines Forschungsprojekts angefallen war. In solchen Fällen kann es ausnehmend mühsam oder logistisch gar unmöglich sein, alle un- tersuchten Personen zu kontaktieren, um sie um die Zusage zu Weiterverwendung von Daten und Proben zu bitten. Dafür wurde der sogenannte «Generalkonsent» (GK) geschaffen, mit dem Patientinnen und Pati-

Zuweilen ist es unmöglich, alle Patienten zu kontaktieren, um sie um die Zusage zur Weiterverwendung von Daten und Proben zu bitten.

enten klinisch Forschenden die Bewilligung respektive Generalabsolution erteilen können, archiviertes Material weiter wissenschaftlich auszuwerten. Die genauen 
Forschungsziele sind im Moment der Zusage meist noch gar nicht definiert. Wie es im Vorspann des Artikels von Sprecher und Talanova zum Verbesserungspotenzial des Generalkonsents zu lesen steht: «Ein broad consent ermöglicht die flexible Nutzung von Daten und Proben zu Forschungszwecken unter Wahrung der Autonomie der Betroffenen» [1]. Ferner kommt es vor, dass prospektive klinische Studien Untersuchungen vorsehen, die ihrerseits Zufallsbefunde ergeben, die klinisch durchaus relevant sein mögen. Zufallsbefunde können bei der Information der Betroffenen naturgemäss nicht einzeln vorweggenommen werden.

Es liegt in der Eigenverantwortung der Betroffenen, ihr Einverständnis aus freien Stücken und eigenem Antrieb aktiv zurückzuziehen.

Die Autorinnen des erwähnten Artikels, beide an einem Institut oder Zentrum für Gesundheitsrecht tätig, bemängeln nun mehrere rechtliche Aspekte des GK. Ich greife zwei Punkte, die die Autorinnen kritisieren, heraus: Der Generalkonsent sei unbegrenzt gültig, und die Patientinnen und Patienten hätten ein Recht auf Nichtwissen (Art. 8 Abs. 2 HFG) von Befunden, die im Rahmen von Untersuchungen anfallen.

\section{Praxisrelevante Aspekte}

Zur Gültigkeitsdauer eines Generalkonsents: Wenn es klinisch Forschenden schon schwerfällt oder gar unmöglich ist, eine initiale Einwilligung zur Weiterverwendung von Daten und asservierten Proben von Betroffenen einzuholen, dann gilt dies mutatis mutandis auch für den Zeitpunkt, zu dem ein befristet erteilter Generalkonsent abgelaufen wäre. Die Patientin respektive der Patient müsste aber im Text des Generalkonsents beraten werden, dass sie oder er aus eigener Initiative die Einwilligung jederzeit zurückziehen kann - dies scheint mir der akzeptable und praktikable Weg. Und so steht es denn auch im GK: «Sie können Ihre Einwilligung jederzeit (...) widerrufen, ohne eine Begründung dafür abzugeben.» Es ist nicht einzusehen, weshalb hier nicht an die Eigenverantwortung der Betroffenen appelliert werden kann, ihr Einverständnis aus freien Stücken und vor allem aus eigenem Antrieb aktiv zurückzuziehen. Die Arbeit der Forschenden wird dadurch wesentlich erleichtert - nur ist es wohl so, dass die geistigen Eltern des HFG diesem Aspekt wenig Bedeutung beimassen und beimessen.

Das Recht auf Nichtwissen ist ein sehr problematischer Aspekt, der aus einer philosophisch-juristischen Sicht propagiert wird, aber praxisfern sein kann. Art. 8 im
HFG hält lapidar fest, dass «die betroffene Person auf diese Information verzichten kann". Er muss meines Erachtens aus der klinischen Praxis ausgelegt werden. Der Verzicht der Testperson auf Information mag angemessen oder mindestens unschädlich sein, wenn es sich um Forschungsdaten handelt, deren klinische Relevanz zum Zeitpunkt einer laufenden Studie unklar ist oder die vielleicht klinisch nie bedeutsam werden Zufallsbefunde, die klinisch wichtig sind, müssen aus ärztlicher Sicht jedoch zwingend mitgeteilt werden, und auch mitgeteilt werden dürfen.

\section{Ethisch problematisch}

Ich erlaube mir eine Ausweitung meiner Kommentare zu diesem Thema auf prospektive Studien (die mit einem GK natürlich nicht abgedeckt werden können), bei denen sich das "Recht auf Nichtwissen» der Teilnehmenden in praxi in noch schwierigerem Licht darstellt als bei der Weiterverwertung bestehender Daten oder Proben. Besteht die Patientin respektive der Patient auf sein Recht auf Nichtwissen, so darf ihm z.B. nach einer MR-Untersuchung des Gehirns, die in einer klinischen Studie mit einer psychiatrischen Fragestellung angeordnet wurde, nicht mitgeteilt werden, dass per Zufall ein klinisch stummer operabler Hirntumor gefunden wurde. Desgleichen kann in einem studienhalber angefertigten Blutbild überraschend eine Leukämie zum Vorschein kommen, bevor sie klinische Symptome zeitigt. Was geschieht, wenn in einer Studie mit «next-generation-sequencing» nebenher eine bislang unbekannte konstitutionelle Genmutation mit erheblichem Krebsprädispositions-Risiko gefunden wird? Der Patientin, die anlässlich der Unterzeichnung der Studieninformation oder des Generalkonsents auf ihr Recht auf Nichtwissen pochte, dürfen diese Informationen nicht weitergegeben werden - ein Verstoss gegen diese Vorgabe würde die Juristen auf den Plan

Was geschieht, wenn in einer Studie nebenher ein erhebliches Krebsprädispositions-Risiko bei einem Patienten gefunden wird?

rufen! So stelle man sich doch den Moment vor, wenn sich die Betroffene später, wenn sie sich schliesslich mit der etablierten Diagnose eines mittlerweile symptomatischen Glioms, einer Leukämie oder eines BRCAPOS Mammakarzinoms konfrontiert sieht, nachfragt, ob diese Befunde nicht schon bei den früheren Studienuntersuchungen hätten aufgedeckt werden können. «Ja», wäre die Antwort der ärztlichen Leitung der Studien, nur hätte die Betroffene in der Studieninformation oder im GK-Formular leider das Feldchen 
«Recht auf Nichtwissen» angekreuzt, und deswegen hätte man sie halt nicht informieren dürfen.

Es gibt nicht nur eine Ethik für Studienteilnehmende oder solche, die es vielleicht werden könnten. Es gibt auch ethische Aspekte, die die Interessen der Prüfärztinnen und -ärzte betreffen. Audiatur et altera pars! Das Recht auf Nichtwissen vom juristischen Schreibtisch aus durchzusetzen, führt aus ethischer Sicht in eine problematische Konfliktsituation für die Prüfärztinnen und -ärzte. Der einzige Ausweg aus dieser Situation ist, systematisch allen Patientinnen und Patienten, die ihr Recht auf Nichtwissen einfordern, die Teilnahme an Forschungsprojekten zu verweigern. Die Autorinnen des Artikels über den Generalkonsent haben diesen praxisrelevanten Aspekt ausser Acht gelassen.

\section{Speditive klinische Forschung ist essenziell}

Generell ist zu sagen, dass die Bevölkerung Fortschritte in der Medizin erwartet, und zwar bisweilen rasch, so aktuell in der medizinischen Bewältigung der "Corona-Krise». Speditive klinische Forschung ist essenziell. So sind denn in den Regularien, die die klini-

\section{Das Wichtigste in Kürze}

- Der Autor befürwortet das momentan gültige Modell des Generalkonsents (GK).

- Die Kritik an der Gültigkeitsdauer des GK und das Recht auf Nichtwissen weist er zurück. Die Betroffenen könnten in Eigenverantwortung jederzeit ihr Einverständnis zur Weiterverwendung von Daten und Proben zurückziehen. Das Recht auf Nichtwissen würde in letzter Konsequenz dazu führen, dass Patientinnen und Patienten, die dieses Recht einfordern, von Forschungsprojekten ausgeschlossen werden müssten, um aus ethischer und rechtlicher Sicht problematische Konfliktsituationen vermeiden zu können.

- Es wird unterstrichen, dass die rechtliche Steuerung klinischer Studientätigkeit eher im Hinblick auf die klinische Forschung denn einer juristisch-puristisch orientierten Politik er- sche Forschung rechtlich steuern, einerseits berechtigte Sicherheitsanliegen der Testpersonen und ihrer Daten oder Proben zu berücksichtigen und andererseits möglichst schlanke Vorgaben zugunsten zügiger Bewilligungsprozesse klinischer Studien aufzustellen. Beides ist sorgfältig gegeneinander abzuwägen. Diese Balance gerät zunehmend in Schieflage, indem die Bewilligungsbedingungen für Forschungsprojekte in teilweise kleinkrämerischer Art immer weiter verfeinert werden; zur Freude der Juristinnen und Juristen, weniger der klinisch Forschenden. Die rechtliche Steuerung klinischer Studientätigkeit sollte schwergewichtig aus dem Blickwinkel der klinischen Praxis und nicht im Sinne einer juristisch-puristisch orientierten Politik ausgeübt werden.

Bemerkung:

Der Autor vertritt im Artikel ausschliesslich seine persönliche Meinung.

Bildnachweis

Symbolbild, ( 18 percentgrey | Dreamstime.com

\section{Literatur}

1 Talanova V, Sprecher F. Verbesserungspotenzial des Generalkonsents. Schweiz. Ärzteztg. 2020;101(38):1197-200.

\section{L'essentiel en bref}

- L'auteur plébiscite le modèle actuel du consentement général (CG).

- II rejette les critiques sur la durée de validité du CG et le droit de ne pas savoir. Les personnes concernées peuvent à tout moment et en toute connaissance de cause retirer leur consentement à la réutilisation de leurs données et des échantillons. Le droit de ne pas savoir conduirait en fin de compte à exclure des projets de recherche les patients qui revendiquent ce droit, ceci afin d'éviter des situations de conflits problématiques d'un point de vue éthique et juridique.

- L'auteur souligne que le pilotage juridique des activités d'essais cliniques doit être effectué dans l'optique de la recherche clinique plutôt que d'une politique à orientation juridico-puriste. 